\title{
KCa3.1 channels are involved in the infiltrative behavior of glioblastoma in vivo
}

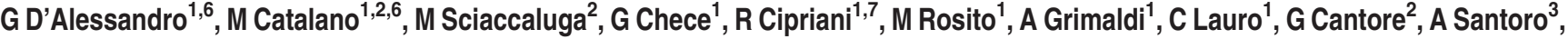 \\ B Fioretti ${ }^{4}$, F Franciolini ${ }^{4}, \mathrm{H}$ Wulff ${ }^{5}$ and C Limatola ${ }^{*, 1,2}$
}

Glioblastoma multiforme (GBM) is a diffuse brain tumor characterized by high infiltration in the brain parenchyma rendering the tumor difficult to eradicate by neurosurgery. Efforts to identify molecular targets involved in the invasive behavior of GBM suggested ion channel inhibition as a promising therapeutic approach. To determine if the $\mathrm{Ca}^{2+}$-dependent $\mathrm{K}^{+}$channel $\mathrm{KCa} 3.1$ could represent a key element for GBM brain infiltration, human GL-15 cells were xenografted into the brain of SCID mice that were then treated with the specific KCa3.1 blocker TRAM-34 (1-((2-chlorophenyl) (diphenyl)methyl)-1H-pyrazole). After 5 weeks of treatment, immunofluorescence analyses of cerebral slices revealed reduced tumor infiltration and astrogliosis surrounding the tumor, compared with untreated mice. Significant reduction of tumor infiltration was also observed in the brain of mice transplanted with KCa3.1-silenced GL-15 cells, indicating a direct effect of TRAM-34 on GBM-expressed KCa3.1 channels. As KCa3.1 channels are also expressed on microglia, we investigated the effects of TRAM-34 on microglia activation in GL-15 transplanted mice and found a reduction of CD68 staining in treated mice. Similar results were observed in vitro where TRAM-34 reduced both phagocytosis and chemotactic activity of primary microglia exposed to GBM-conditioned medium. Taken together, these results indicate that $\mathrm{KCa} .1$ activity has an important role in GBM invasiveness in vivo and that its inhibition directly affects glioma cell migration and reduces astrocytosis and microglia activation in response to tumor-released factors. KCa3.1 channel inhibition therefore constitutes a potential novel therapeutic approach to reduce GBM spreading into the surrounding tissue. Cell Death and Disease (2013) 4, e773; doi:10.1038/cddis.2013.279; published online 15 August 2013

Subject Category: Cancer

Glioblastoma multiforme (GBM) is the most common and malignant primary brain tumor. Despite continuous progress in neurosurgery, its infiltrative behavior precludes complete tumor resection and is certainly the main reason of the poor clinical outcome for patients., ${ }^{1,2}$ The invasion of cerebral parenchyma by GBM cells is mediated by membrane and soluble factors released by malignant cells, which contribute to extracellular matrix digestion and neuron excitotoxicity. ${ }^{3-6}$ This process is boosted by the interaction with local (microglia) and infiltrating immune cells (macrophages and Treg cells), which produce cytokines and matrix-degrading enzymes important for tumor growth and expansion into the brain. ${ }^{7,8}$ The chemokine CXCL12 and its receptor CXCR4 are major factors contributing to the degree of glioma malignancy and invasiveness, ${ }^{9-11}$ and it has been proposed that the so called secondary structures of Scherer might be formed by the strategic expression of CXCL12 and CXCR4 at the leading edge of invading cells. ${ }^{12}$ We have recently demonstrated that CXCL12 induces the activation of $\mathrm{Ca}^{+}$-dependent intermediate-conductance $\mathrm{K}^{+}$channels (KCa3.1) and that pharmacologic block of these channels leads to a selective inhibition of CXCL12-induced GBM migration in vitro. ${ }^{13}$ Other ion channels like large-conductance $\mathrm{Ca}^{+}$-dependent $\mathrm{K}^{+}$ channels (KCa1.1 or BK), and voltage-gated $\mathrm{Cl}^{-}$channels modulate the migration of GBM cells ${ }^{14-17}$ and are involved in the continuous changes of cell shape and volume required for their migration in the narrow extracellular spaces created by the above described mechanisms.

In the normal adult brain, KCa3.1 channels are primarily expressed in microglia ${ }^{18}$ and are mostly undetectable in other

\footnotetext{
${ }^{1}$ Institute Pasteur, Cenci Bolognetti Foundation, Department of Physiology and Pharmacology, Sapienza University of Rome, Rome, Italy; ${ }^{2}$ IRCCS Neuromed, Pozzilli, Italy; ${ }^{3}$ Department of Neurology and Psychiatry, Sapienza University of Rome, Rome, Italy; ${ }^{4}$ Department of Cellular and Environmental Biology, Perugia University, Perugia, Italy and ${ }^{5}$ Department of Pharmacology, University of California, Davis, CA, USA

*Corresponding author: C Limatola, Department of Physiology and Pharmacology, Sapienza University of Rome, Piazzale Aldo Moro 5, Rome 00185, Italy. Tel: + 39064969 0243; Fax: + 39064991 0851; E-mail: cristina.limatola@uniroma1.it

${ }^{6}$ These authors contributed equally to this work.

${ }^{7}$ Present address: Departamento de Neurociencias, Universidad del Paıs Vasco, (UPV/EHU), Vizcaya, Spain

Keywords: $\mathrm{Ca}_{2}{ }^{+}$-dependent $\mathrm{K}^{+}$channels; glioblastoma; invasion; astrocytes; microglia

Abbreviation: KCa3.1, intermediate-conductance calcium-dependent potassium channel 1; TRAM-34, 1-[(2-chlorophenyl)(diphenyl)methyl]-1H-pyrazole; GBM, glioblastoma multiforme; SCID, severe combined immunodeficiency; KCa1.1, large-conductance calcium-dependent potassium channel 1; shRNA, short hairpin ribonucleic acid; DMEM, Dulbecco's modified minimum essential medium; FBS, fetal bovine serum; EGF, epidermal growth factor; RT-PCR, reverse transcriptase polymerase chain reaction; GFAP, glial fibrillary acidic protein; IPTG, isopropyl-beta-D-1-thiogalactopyranoside; MOPS, 3-(N-morpholino)propanesulfonic acid; EGTA-K, ethylene glycol tetraacetic acid- potassium; KCa2, Small-conductance calcium-activated potassium channel; BSA, bovine serum albumin; HEPES, hydroxyethyl piperazineethanesulfonic acid; DMSO, dimethyl sulfoxide; LPS, lipopolysaccharides; PBS, potassium phosphate buffer; cDNA, complementary deoxyribonucleic acid; LC/MS, liquid chromatography-mass spectrometry; HPLC, high-performance liquid chromatography; ANOVA, analysis of variance; siRNA, small interfering ribonucleic acid; MMP, matrix metalloproteinases; M-CSF, macrophage-colony stimulating factor; TEA, tetraethylammonium; NS309, 6,7-dichloro-1H-indole-2,3-dione 3-oxime Received 06.5.13; revised 25.6.13; accepted 02.7.13; Edited by G Raschellá
} 
cell populations. ${ }^{19}$ In contrast, KCa3.1 channels are functionally expressed in GBM cell lines and biopsy samples. ${ }^{20-22}$ One of the first reported inhibitors of $\mathrm{KCa} 3.1$, clotrimazole, has been found to sensitize glioma cells to radiotherapy by arresting cell cycle in $\mathrm{G} 1 .{ }^{23-25}$ However, clotrimazole cannot be used clinically because it is a strong inhibitor of cytochrome P450-dependent enzymes and induces liver toxicity. The clotrimazole derivate TRAM-34 (1-((2-chlorophenyl)diphenylmethyl)-1H-pyrazole), in contrast, is free of these toxic effects $^{26}$ and has been used in in vivo models of pathologies such as vascular restenosis, ${ }^{27}$ ischemic stroke, ${ }^{28} \mathrm{EAE},{ }^{29}$ tumor angiogenesis, ${ }^{30}$ atherosclerosis, ${ }^{31}$ and asthma. ${ }^{32}$

In the present work, we xenografted severe combined immunodeficiency (SCID) mice with human GL-15 GBM cells and evaluated the effect of blocking $\mathrm{KCa} 3.1$ channels with TRAM-34 or their silencing by shRNA on tumor cells infiltration in the brain. These glioma cells were chosen because their infiltrative behavior makes them an ideal experimental system for studying compounds affecting tumor spreading in the brain parenchyma. ${ }^{33}$ We observed that targeting $\mathrm{KCa} 3.1$ induced significant reductions of the tumorinfiltrated area and reduced the maximal spreading of GBM in the cerebral parenchyma. We also demonstrated that $\mathrm{KCa} 3.1$ inhibition decreased astrogliosis and microglia/macrophages activation at the boundary of the tumor and suppressed microglia phagocytosis and migration toward GBMconditioned medium in vitro.

\section{Results}

TRAM-34 reduces $\mathrm{GBM}$ infiltration in vitro and in vivo. We previously demonstrated that TRAM-34 is effective in reducing CXCL12-induced GBM migration in cell lines and primary cells obtained from patients. ${ }^{13}$ We now first performed preliminary in vitro experiments to investigate the effect of KCa3.1 inhibition on CXCL12-induced Matrigel invasion by GL-15 cells, and the results obtained confirmed that CXCL12- $(100 \mathrm{nM}, 18 \mathrm{~h})$ stimulated cell infiltration (C: $9.90 \pm 0.54$ cells/field; CXCL12: $18.44 \pm 0.81$ cells/field, $n=4, \quad P<0.001)$ was significantly inhibited by TRAM-34 $(5 \mu \mathrm{M})$ treatment (TRAM-34: $8.84 \pm 0.48$ cells/field; CXCL12/ TRAM-34: $9.45 \pm 0.41$ cells/field, $n=4)$.

To investigate if KCa3.1 channel inhibition was also able to reduce GBM infiltration in vivo, GL-15 cells were injected in SCID mouse brains and animals were treated with TRAM-34 or vehicle (peanut oil) for 5 weeks as described in the methods section. Under these conditions, TRAM-34 reached pharmacologically active levels: ${ }^{26}$ in fact, $2 \mathrm{~h}$ after the last injection, brain levels of TRAM-34 were $1367 \pm 201 \mathrm{nM}$ and were maintained at $424 \pm 39 \mathrm{nM}$ after $12 \mathrm{~h}$ (Table 1). We further analyzed brain slices for tumor infiltration into the brain parenchyma. Figure 1 shows representative images of brain slices stained for human nuclei from mice treated with peanut oil (Figure 1a and c) or TRAM-34 (Figures 1b and d). These results show a significant reduction of tumor-infiltrated area $(43.2 \%$ reduction) in mice treated with TRAM-34 in comparison with vehicle-treated mice $(0.074 \pm 0.007 \%$ and $0.042 \pm 0.006 \%$, respectively, $n=6, P<0.05$, Figure $1 e)$, demonstrating that $\mathrm{KCa} 3.1$ channel inhibition results in a reduced GBM spreading in the brains of xenografted mice.
Table 1 TRAM-34 concentration in mouse brains

\begin{tabular}{lc}
\hline Time (h) & TRAM-34 (nM) \\
\hline 0 & 0 \\
2 & $1367 \pm 201$ \\
12 & $424 \pm 39$ \\
\hline
\end{tabular}

SCID mice ( $n=3$ for each time point) were injected with GL-15 cells as described in the text and treated for 5 weeks with TRAM-34. At different time points after the last treatment, mice were sacrificed, the brains were isolated, and analyzed using HPLC/MS as described in the text.

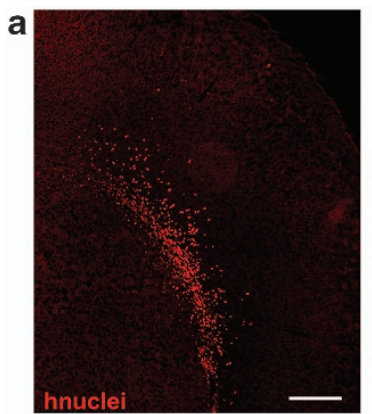

C

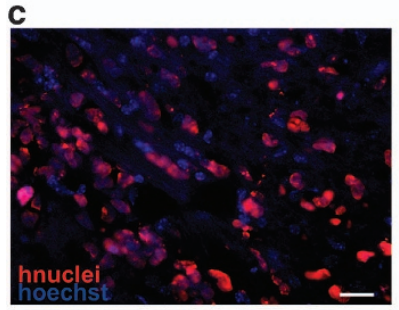

e

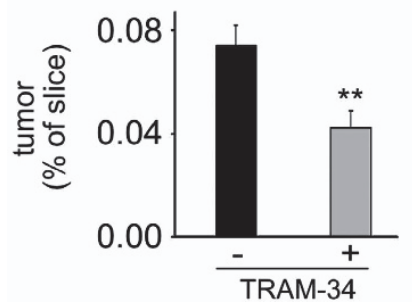

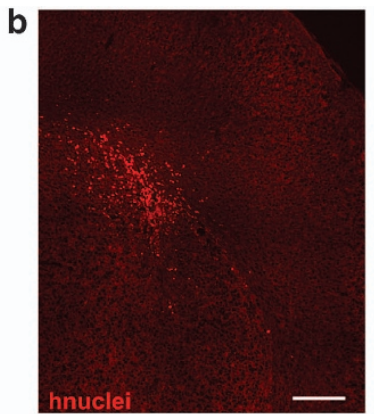

d

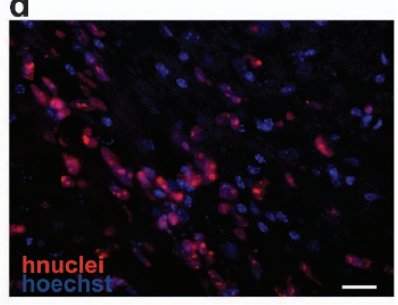

f

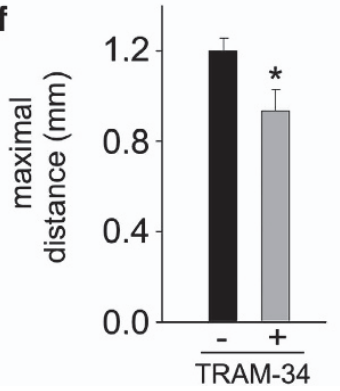

Figure 1 TRAM-34 reduces tumor-infiltrated area and maximal migration distance of GL-15 in the mouse brain. Brain slices stained with anti human nuclei (hnuclei, in red) in vehicle- (a, c) and TRAM-34- (b, d) treated mice. $\mathbf{a}$ and $\mathbf{b}$ show the infiltrated (right) hemisphere $(\times 10)$; $\mathbf{c}$ and $\mathbf{d}$ are magnifications $(\times 40$; total nuclei: blue, Hoechst) of a part of the areas indicated in $\mathbf{a}$ and $\mathbf{b}$; (e) quantification of tumor-infiltrated area (area occupied by human nuclei-positive cells versus total slice area) in vehicle- and TRAM-34-treated mice ( ${ }^{* *} P<0.001$, Student's $t$-test). (f) brain slices were analyzed for the presence of GL-15 cells (cells positive for human nuclei staining) in the sagittal plane. Values are the maximal distances (in $\mathrm{mm})$ obtained in vehicle- $(n=6)$ and TRAM-34-treated mice $(n=6) .{ }^{*} P<0.05$, Student's t-test. Bars: a, b: $225 \mu \mathrm{m} ; \mathbf{c}, \mathbf{d}$ : $10 \mu \mathrm{m}$

GBM cells are known to preferentially migrate along well described pathways in the brain. ${ }^{33-35}$ We here confirmed this observation and found that injected GBM cells always invaded the brain moving along the external capsula region (see representative Figure 1a). To evaluate the maximal distance 
reached by tumor cells in our experimental model, we measured the extent of GBM infiltration along the sagittal brain axis. As represented in Figure 1f, TRAM-34-treated mice showed a significantly reduced cell migration from the point of injection. Collectively, the maximal distance (in the sagittal plane) covered by GBM in vehicle-treated mice was $1.203 \pm 0.051 \mathrm{~mm}$, whereas it was reduced to $0.930 \pm 0.096 \mathrm{~mm}$ in the brain of TRAM-34-treated mice $(n=6 ; P<0.05)$

KCa3.1 silencing by specific shRNA infection reduced GBM infiltration in xenografted mouse brains. KCa3.1 channels are expressed by GBM but are also found in microglia and endothelial cells in the brain. ${ }^{36,37}$ To investigate whether the effect of $\mathrm{KCa} 3.1$ inhibition on tumor dispersion in vivo was due to the blockage of these channels specifically on GBM cells, we injected GL-15 cells silenced for KCa3.1 expression and compared infiltration of these cells in the brains of xenografted mice. Preliminarily, we tested two IPTG-inducible shRNA constructs raised against KCa3.1 mRNA for their ability to silence KCa3.1 expression and function in the infected cells. Results obtained by semiquantitative PCR analysis revealed a significant silencing of KCa3.1 mRNA only with one construct (GL-15-shRNA1), with $75.4 \pm 7.5 \%$ reduction of mRNA presence in the IPTGinduced clone (Figure 2a). These cells were also tested for KCa3.1 channel activity: electrophysiological recordings performed on vehicle- and IPTG-induced cells demonstrated that NS309 $(10 \mu \mathrm{M})$, a potent activator of KCa3.1, induced a typical KCa3.1 current in control cultures but not in IPTGtreated cultures (Figure $2 \mathrm{~b}$, left). In detail, in control cells a $\mathrm{K}^{+}$-selective, voltage-independent current was evoked, which was greatly inhibited by TRAM-34 (Figure 2b, left). The TRAM-34-sensitive current density, assessed at $0 \mathrm{mV}$ to reduce the interference from $\mathrm{Cl}^{-}$currents, was $6.0 \pm 0.3$ $\mathrm{pA} / \mathrm{pF}(n=3)$, in line with previous reports. ${ }^{13,21}$ Greatly reduced currents were by contrast observed when the same experimental conditions and stimulating protocols were applied to IPTG-induced cells (Figure 2b, right). The TRAM-34-sensitive current density assessed at $0 \mathrm{mV}$ was $0.9 \pm 0.3 \mathrm{pA} / \mathrm{pF}(n=4)$. To verify the effectiveness of KCa3.1 silencing with a functional assay, these cells were tested for their chemotaxis toward CXCL12 or EGF gradients. Under these conditions, we observed a selective lack of migration toward CXCL12 ( $n=3-5$, Figure 2 c), in accordance with our previous data demonstrating a selective involvement of KCa3.1 in CXCL12-induced chemotaxis. ${ }^{13}$

We then xenografted GL-15-shRNA1 cells $\left(4 \times 10^{5}\right.$ cells/ mice) into 14 SCID mice and induced shRNA expression in seven of them by adding IPTG $(10 \mathrm{mM})$ twice a week in drinking water. ${ }^{38,39}$ The remaining mice received normal water. After 5 weeks, all the mice were sacrificed and brains were analyzed for GBM infiltration. As reported in Figure 2d, IPTG-induced mice showed a specific reduction of mean infiltrated brain area in comparison with un-induced mice (respectively, $0.078 \pm 0.008 \%$ and $0.12 \pm 0.01 \%, P<0.05$ ) Immunofluorescence analysis of these brain slices confirmed that immunoreactivity for $\mathrm{KCa} 3.1$ was much less evident in IPTG-treated mice (Figure 2e), consistent with data obtained in vitro (Figures $2 \mathrm{a}-\mathrm{C}$ ).

Blockade of KCa3.1 channels decreased astrocyte and microglia activation in the brains of GBM-xenografted mice. To investigate whether the reduction of tumor infiltration in the brains of TRAM-34-treated mice was accompanied by a reduction of astrocyte activation, ${ }^{40}$ brain slices obtained from TRAM-34- or vehicle-treated mice were analyzed for GFAP expression. Figure 3a shows representative images of GFAP expression in slices obtained from vehicle- (left) and TRAM-34- (right) treated mice. Figure $3 b$ reports the mean values of GFAP staining (as the ratio of positively stained area for GFAP per slice area) in slices obtained from vehicle-(0.389 $\pm 0.055 \%, n=6)$ and TRAM-34-treated mice $(0.169 \pm 0.028 \%, n=6)$, corresponding to a reduction of
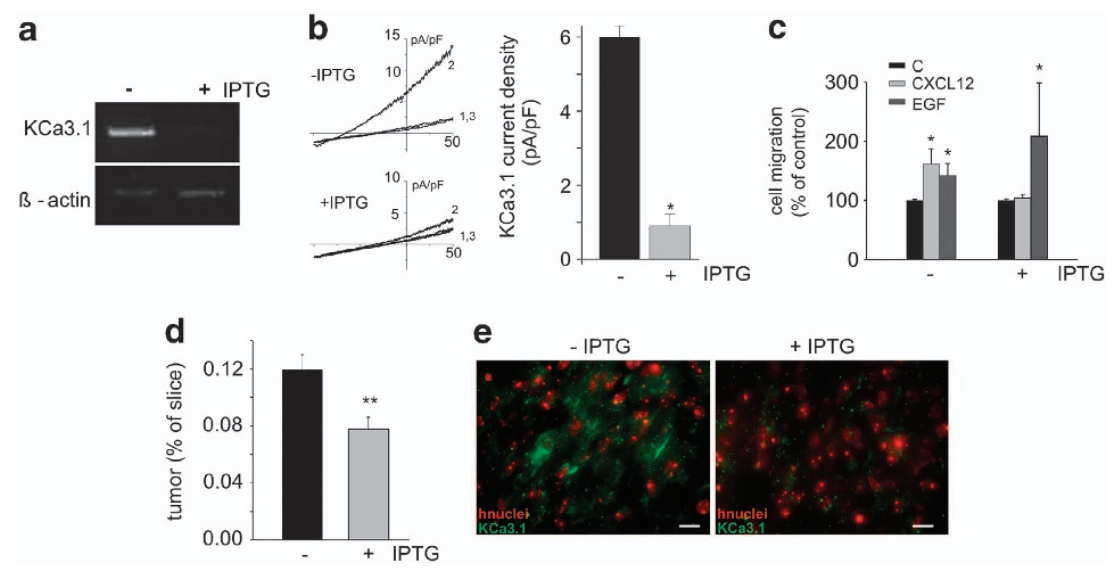

Figure $2 \mathrm{KCa} 3.1$ shRNA-silenced GL-15 cells show reduced infiltration in the mouse brain. (a) mRNA quantification of KCa3.1 relative to $\beta$-actin in control and IPTGinduced cells; (b) left, representative I-Vs from control and IPTG-induced cells, in C (1), NS309 (10 $\mu \mathrm{M})$ (2), and NS309/TRAM-34 (3 $\mu \mathrm{M})(3)$. Right, bar plot showing the TRAM-34-sensitive KCa3.1 current density in control and IPTG-induced cells ${ }^{*} P<0.05$; (c) control and IPTG-treated cell migration toward CXCL12 or EGF $\left({ }^{\star} P<0.05\right.$ versus $C$ of the same experimental group, Student's t-test); (d) quantification of tumor-infiltrated area in the brain of mice injected with shRNA-silenced GL-15 cells (area occupied by human nuclei-positive cells versus total slice area) and treated or not with IPTG ${ }^{* *} P<0.01$, Student's $t$-test); (e) immunofluorescence analysis of KCa3.1 expression (green; hnuclei: red) in brain slices obtained from mice injected with shRNA-silenced GL-15 cells un-induced (left) or induced (right) with IPTG. Bars, $10 \mu \mathrm{m}$ 
a
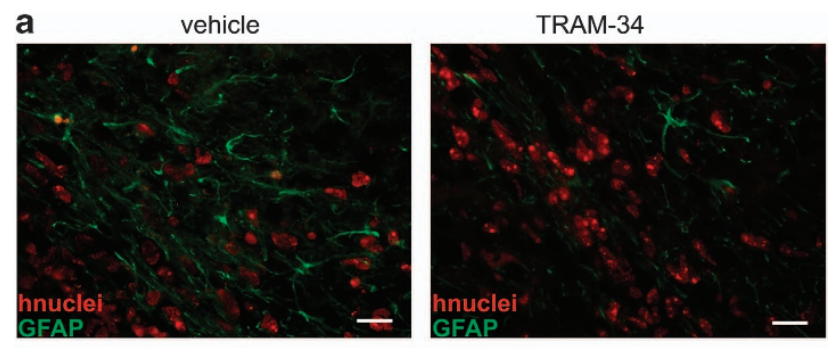

b

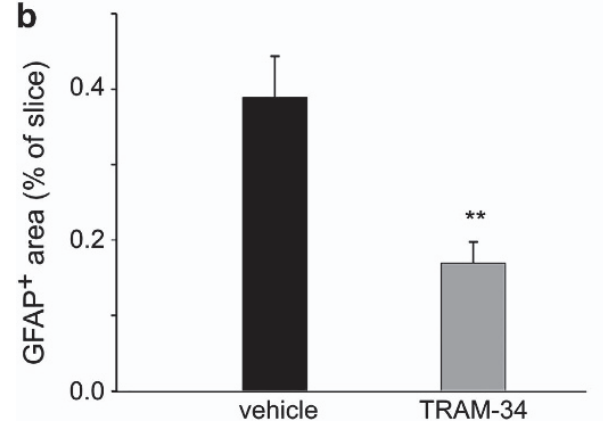

Figure 3 TRAM-34 reduces astrogliosis. (a) representative immunofluorescences of GFAP (green) and human nuclei (red) in vehicle- and TRAM-34- treated mice ( $\times 40$; bars $10 \mu \mathrm{m})$; (b) quantification of astrogliosis (area occupied by GFAPpositive cells versus total slice area) in vehicle- and TRAM-34-treated mice ${ }^{* \star} P<0.001$, Student's $t$-test)

about $57 \%(P<0.001)$. As previously reported, ${ }^{39}$ the Spearman rank order correlation $(\rho=0.224, n=12 ; P<0.006)$ also revealed a significant correlation between tumor size and GFAP expression in our experimental system.

Microglia activation was investigated by staining for CD68, a microglia/macrophage marker of phagocytic activity. Data reported in Figure 4a show representative images of CD68 expression in slices obtained from vehicle- (left) and TRAM-34- (right) treated mice, and Figure $4 \mathrm{~b}$ shows the mean values of CD68 staining per slice, demonstrating a significant difference between the two populations (vehicle: $0.027 \pm 0.002 \%$; TRAM: $0.017 \pm 0.002 \%, P<0.001, n=4$ ), with a $38 \%$ mean reduction. To further validate these results and to verify whether KCa3.1 channel activity on microglia was important for microglia-GBM interactions, we performed in vitro experiments to investigate whether TRAM-34-treated microglia cells had different phagocytic activity when exposed to GBM-conditioned medium. Data reported in Figure 4c indicate that TRAM-34 treatment significantly reduced the phagocytic activity of primary microglia exposed to different GBM-conditioned media (GL-15 and MZC cells, a primary GBM cell line with electrophysiological responses similar to GL-15 cells, see Ruggieri et al. ${ }^{41}$ ). ${ }^{* \star} P<0.01$ for $\mathrm{C}$ of GBM-treated versus $C$ of untreated microglia; ${ }^{\# \#} P<0.01$ for TRAM versus $C$ of GBM-treated microglia, for both cell lines $(n=3-5)$. These results indicate that microglia activation near the brain-infiltrated region requires $\mathrm{KCa} 3.1$ channel activity.

We also investigated microglia migration toward GBMconditioned medium: results shown in Figure 4d indicate that microglia migration induced by GBM-(GL-15 and $M Z C)$ conditioned medium ( $\mathrm{C}$ of GBM-treated versus $\mathrm{C}$ of nil treated microglia, ${ }^{* *} P<0.01$ ) was strongly inhibited by TRAM-34 treatment (TRAM versus $\mathrm{C}$ of GBM-treated microglia, ${ }^{\# \#} P<0.01$ for both cell lines; $\left.n=5-6\right)$. These in vitro data are in agreement with the CD68 staining obtained in slices and indicate reduced microglia recruitment and activation when KCa3.1 channel activity is inhibited.

\section{Discussion}

In this study, we demonstrated that KCa3.1 channels are involved in modulating GBM cell infiltration in the brain parenchyma in vivo, directly acting on tumor cell movement and also modulating the recruitment of active microglia at the tumor boundaries with effects on tumor spreading and astrogliosis.

The role of ion channels in GBM migration has received wide attention in recent years, with several reports demonstrating their key role in modulating, in concert with aquaporins, tumor cell shape and volume and maintaining a physiological cytoplasmic osmolarity. ${ }^{42}$ In particular, KCa3.1 channels have been demonstrated to be involved in modulating GBM migration in vitro in response to $\mathrm{CXCL} 12,{ }^{13}$ serum, ${ }^{43}$ and bradykinin. ${ }^{17}$ The importance of KCa3.1 for GBM invasion of cerebral tissue has been recently demonstrated in brain slices transplanted with GBM. ${ }^{17}$ All these data are in agreement with our results, demonstrating for the first time the in vivo effect of inhibiting KCa3.1 channels on GBM spreading in the brain parenchyma. In particular, we demonstrated a direct involvement of $\mathrm{KCa} 3.1$ channels in GBM invasion, showing that their specific silencing in tumor cells significantly reduced the cerebral area occupied by tumor in xenografted mice. Given the involvement of other $\mathrm{K}^{+}$ channels (KCa1.1) in the modulation of GBM cell movement, ${ }^{22}$ it is of course possible that even greater inhibition of cell invasion might be obtainable when both channels are blocked.

We further cannot exclude the possibility that the reduction of tumor infiltration observed in TRAM-34-treated mice might be partly due to effects of the drug on cell proliferation, as TRAM-34 inhibition of KCa3.1 has been shown to reduce GBM cell growth in vitro. ${ }^{44}$ However, the $\mathrm{IC}_{50}$ for this effect is much higher $(14 \mu \mathrm{M})$ than that required for current inhibition $(70 \mathrm{nM}),{ }^{21}$ and the same authors show that siRNA down regulation of $\mathrm{KCa} 3.1$ channels is ineffective on GBM cell proliferation, ${ }^{44}$ thus, suggesting possible non-specific effects. This fact, together with our observation that the concentrations of TRAM-34 reached in the brains of treated mice are around $1 \mu \mathrm{M} 2 \mathrm{~h}$ after the last treatment, would discourage this interpretation.

KCa3.1 channels are also expressed by other resident or infiltrating cell types, especially microglia and macrophages. ${ }^{45}$ We therefore considered the possibility that the reduction of GBM infiltration observed in xenografted mice treated with TRAM-34 could be caused in part by effects on cells other than GBM. For example, KCa3.1 channels are involved in several aspects of microglia activation like respiratory burst, migration, cytokines production, and microglia-mediated neuronal killing. ${ }^{35,45-47}$ Indeed, we demonstrated in this paper that microglia/macrophage activation (evidenced by CD68 staining) is reduced in mice treated with TRAM-34, in agreement with results reported in a model of reperfusion 

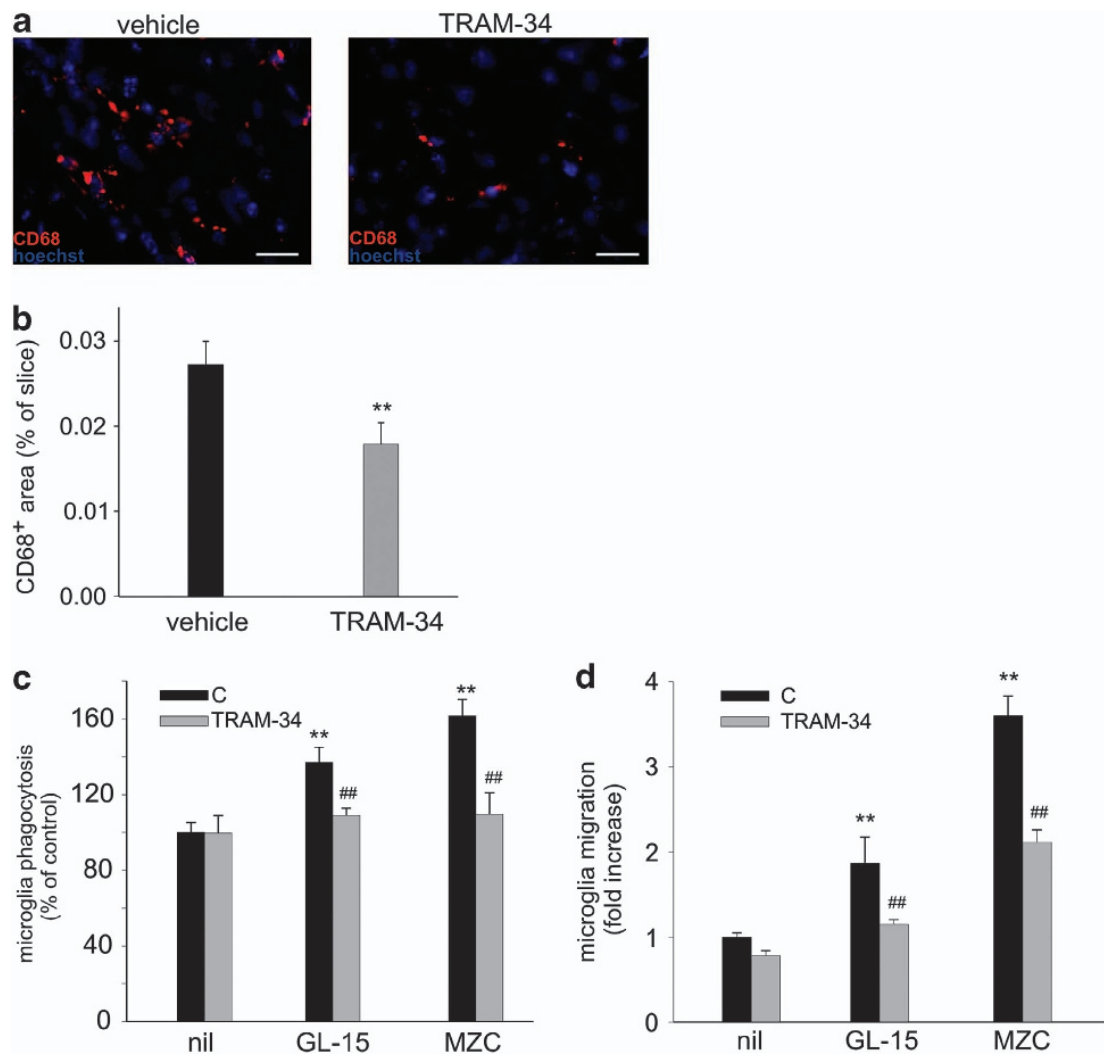

Figure 4 TRAM-34 treatment reduces microglia activation and migration toward GBM. Brain slices obtained from mice xenografted with GL-15 GBM were analyzed for CD68 immunoreactivity (red; total nuclei: blue, Hoechst) in vehicle- and TRAM-34-treated mice (a, bars $10 \mu \mathrm{m}$ ). Mean values of CD68 staining per slice are reported in (b). Data are expressed as the ratio between the area occupied by CD68 positive cells versus total slice area and are the mean of 12 slices per mouse $(n=4)$ for each condition. (c) In vitro phagocytosis of fluorescent FluoSpheres by microglia exposed for $18 \mathrm{~h}$ to control medium (nil) or media conditioned ( $24 \mathrm{~h}$ ) by GL-15 and MZC cells in the presence or in the absence of TRAM-34 $(5 \mu \mathrm{M})$. Only cells phagocyting at least three beads were scored as positive and results are expressed as $\%$ of control $\left(n=3-5\right.$; $\left.{ }^{* *} P<0.001\right)$. (d) In vitro migration of microglia toward control medium (nil) or media conditioned ( $24 \mathrm{~h})$ by GL-15 and MZC cells in the presence or in the absence of TRAM-34 (5 $\mu \mathrm{M})$. Migration is expressed as fold increase in the number of migrating cells in comparison with C, nil-exposed cultures and results are the mean \pm S.E.M. of 5-6 independent experiments

stroke in rats in which TRAM-34 treatment reduced microglia activation and infarct size. ${ }^{28}$ Our in vivo observation was confirmed in vitro by the finding that the phagocytic activity of microglia exposed to media conditioned by different GBM cell lines was strongly reduced by TRAM-34. It is reported that glioma switches the infiltrating macrophages/microglia toward an immunosuppressive, M2-like phenotype: tumor infiltrating macrophages remain in the tumor at higher concentrations than in the surrounding parenchyma, have reduced phagocytic activity, produce less inflammatory cytokines and secrete MMP enzymes promoting GBM invasion. ${ }^{48-50}$ A recent report described an inhibitory effect of factors released specifically by glioma cancer stem cells on monocytes (obtained from peripheral blood) phagocytosis. ${ }^{51}$ However, in the first phase of GBM-microglia interaction, GBM produce factors that attract and activate microglia, like M-CSF. 8,52 Our results suggest that $\mathrm{KCa} 3.1$ activity might be important at this early stage of microglia-GBM interaction, reducing microglia activation and migration toward the tumor, thus, counteracting microglia/macrophages recruitment, which would favor tumor growth and dissemination.

We further demonstrated that TRAM-34-treated mice had reduced astrogliosis, evidenced by reduced GFAP staining.
We would exclude a direct effect of TRAM-34 on astrocytes because these cells do not express $\mathrm{KCa} 3.1$ (no visible KCa3.1 staining on astrocytes in our study) ${ }^{28}$, even if contrasting results were recently reported on spinal cord-activated astrocytes. ${ }^{53}$ Our data are in agreement with other studies reporting a correlation between GBM mass and astrocytic GFAP staining ${ }^{40}$ and also congruent with the hypothesis that the presence of GFAP-positive astrocytes near nestin positive stem-like glioma cells could underlie paracrine interactions between astrocytes and tumor cells. ${ }^{54}$ It remains to be determined whether the reduced astrogliosis is a consequence of the reduced GBM infiltration or microglia/macrophage activation, and how $\mathrm{KCa} 3.1$ inhibition could alter astrocytes-GBM interaction.

In conclusion, our results underscore the potential complexity of inhibiting KCa3.1 channels as a therapeutic intervention against GBM, with both direct and indirect effects on tumor cells, but ultimately resulting in a reduced cerebral infiltration by tumor worthy of further consideration.

\section{Materials and Methods}

Materials. Cell culture medium (Dulbecco's modified minimum essential medium, DMEM), fetal bovine serum (FBS), penicillin G, streptomycin, glutamine, 
sodium pyruvate, recombinant human EGF, red fluorescent FluoSpheres $(1 \mu \mathrm{m}$ diameter), Thermo Script RT-PCR System and Hoechst 33342 were from GIBCO Invitrogen (Carlsbad, CA, USA); recombinant human CXCL12 was from Peprotech (London, UK); PCR reactions were from New England Biolabs (Ipswich, MA, USA); an anti-human antigen nuclei antibody was from Millipore Chemicon (Billerica, MA, USA); an anti-GFAP antibody was from Novus Biological (Littleton, CO, USA); an anti-CD68 was from AbD Serotec (Oxford, UK); a rabbit anti-KCa3.1 antibody was from Santa Cruz Biotech. (Santa Cruz, CA, USA); the 'Absolutely RNA miniprep Kit' was from Stratagene (Santa Clara, CA, USA); IPTG (isopropyl-beta-D-1-thiogalactopyranoside) was from Ambion (Grand Island, NY, USA); NS309 was from Tocris (Bristol, UK); pLKO.1 lentiviral shRNA clones targeting human KCa3.1 mRNA were from SIGMA TRC shRNA library (Oligo ID: TRCN0000322968-04393); matrigel was from BD (Franklin Lakes, NJ, USA); DMSO, secondary antibodies, peanut oil, and all other chemicals were from Sigma-Aldrich (St. Louis, MO, USA) or Pierce (Rockford, IL, USA). TRAM-34 was synthesized as previously described. ${ }^{25}$

Animals and cell lines. Experiments described in the present work were approved by the Italian Ministry of Health in accordance with the guidelines on the ethical use of animals from the European Community Council Directive of 24 November 1986 (86/609/EEC). We used C57BL/6 mice from Jackson Laboratories (Bar Harbor, ME, USA) and CB-17//crHanHsd-Prkdcscid SCID mice from Harlan Laboratories (Correzzana, Italy).

GL-15 and MZC GBM cell lines were cultured in DMEM supplemented with 10\% heat-inactivated FBS, $100 \mathrm{IU} / \mathrm{ml}$ penicillin G, $100 \mu \mathrm{g} / \mathrm{ml}$ streptomycin, $2.5 \mu \mathrm{g} / \mathrm{ml}$ amphotericin $\mathrm{B}, 2 \mathrm{mM}$ glutamine, and $1 \mathrm{mM}$ sodium pyruvate. Cells were grown at $37^{\circ} \mathrm{C}$ in a $5 \% \mathrm{CO}_{2}$-humidified atmosphere. Medium was changed twice a week, and the cells were subcultivated when confluent. MZC cells were kindly provided by Dr. Antonietta Arcella, Neuromed, Italy.

Microglial cultures. Microglia cultures were obtained from mixed glia cultures derived from the cerebral cortices of post natal day $0-1$ ( $p 0-p 1)$ C57BL/6 mice. Cortices were chopped and digested in $15 \mathrm{U} / \mathrm{ml}$ papain for $20 \mathrm{~min}$ at $37^{\circ} \mathrm{C}$. Cells $\left(5 \times 10^{5}\right.$ cells $\left./ \mathrm{cm}^{2}\right)$ were plated on flasks coated with poly-L-lysine $(100 \mu \mathrm{g} / \mathrm{ml})$ in DMEM supplemented with $10 \% \mathrm{FBS}, 100 \mathrm{U} / \mathrm{ml}$ penicillin, and $0.1 \mathrm{mg} / \mathrm{ml}$ streptomycin. After 7-9 days, cells were shaken for $2 \mathrm{~h}$ at $37^{\circ} \mathrm{C}$ to detach and collect microglial cells. These procedures gave almost pure microglial cell populations as previously described. ${ }^{55}$

KCa3.1 silencing by lentiviral transduction of shRNA constructs. GL-15 cells were infected by lentivirus directing IPTG-inducible expression of KCa3.1 shRNA. Cells $\left(1.6 \times 10^{4}\right)$ were plated in 96-well plates and infected for $24 \mathrm{~h}$ according to the manufacturer's instructions. Transduced cells were selected with $2.5 \mu \mathrm{g} / \mathrm{ml}$ puromycin for $3-12$ days. IPTG $(5 \mathrm{mM})$ was added to culture medium to induce shRNA expression. Knockdown efficiency of KCa3.1 channels was evaluated by PCR, electrophysiological recordings, immunofluorescence, and chemotaxis assay.

Electrophysiology. The dialyzed whole-cell patch-clamp configuration was used for electrophysiological recordings. The external solution contained (in $\mathrm{mM}$ ) $140 \mathrm{NaCl}, 5 \mathrm{KCl}, 2 \mathrm{CaCl}_{2}, 2 \mathrm{MgCl}_{2}, 5 \mathrm{MOPS}$, and 20 glucose, $\mathrm{pH}$ 7.4; the internal solution contained (in mM) $150 \mathrm{KCl}, 1 \mathrm{MgCl}_{2}, 5 \mathrm{MOPS}$, and 1 EGTA-K, pH 7.25, and $300 \mathrm{nM}$ free $\mathrm{Ca}^{2+}$ calculated using the free WebMax software (www. stanford.edu/ cpatton/webmaxcS). Octanol $(1 \mathrm{mM})$ was added to the external solution to block gap junctions ${ }^{20}$ and TEA $(3 \mathrm{mM})$ was used to block KCa1.1 channels. KCa2 channels, whose mRNAs are present in GL-15, are not functionally expressed in GL-15 cells. ${ }^{13} \mathrm{GL}-15$ do not express detectable Kv currents. ${ }^{56}$ Membrane capacitance was assessed by using the Membrane Test routine of the pClamp software (Molecular Device, Sunnyvale, CA, USA). Currents were elicited by applying linear voltage ramps from -100 to $+100 \mathrm{mV}$, from a $\mathrm{Vh}$ of $0 \mathrm{mV}$, repeated every $5 \mathrm{~s}$. The KCa3.1 current was estimated as the NS309activated, TRAM-34-sensitive current.

Chemotaxis and invasion assays. CXCL12- or EGF-induced chemotaxis was investigated in the glioblastoma cell line GL-15. Semiconfluent cells were trypsinized, preincubated in chemotaxis medium (DMEM without glutamine, $100 \mathrm{IU} / \mathrm{ml}$ penicillin G, $100 \mu \mathrm{g} / \mathrm{ml}$ streptomycin, $0.1 \% \mathrm{BSA}$, and $25 \mathrm{mM} \mathrm{HEPES}, \mathrm{pH}$ $7.4)$ for $15 \mathrm{~min}$, and plated $\left(7 \times 10^{4} \mathrm{cells} / \mathrm{cm}^{2}\right)$ on poly-L-lysine-coated transwells ( $8 \mu \mathrm{m}$ pore size filters) in this same medium. The lower chamber contained
CXCL12 $(50 \mathrm{nM}), \mathrm{EGF}(100 \mathrm{ng} / \mathrm{ml})$ or vehicle. After $3 \mathrm{~h}$ of incubation at $37^{\circ} \mathrm{C}$, cells were treated with ice-cold $10 \%$ trichloroacetic acid for $10 \mathrm{~min}$. Cells adhering to the upper side of the filter were scraped off, whereas cells on the lower side were stained with a solution containing $50 \%$ isopropanol, $1 \%$ formic acid, and $0.5 \%$ (wt/vol) brilliant blue R 250. Stained cells were counted in more than 20 fields with a $\times 40$ objective. For microglia chemotaxis, the medium alone or conditioned for $24 \mathrm{~h}$ by GL-15 or MZC cells was used as chemoattractant in the bottom well $(3 \mathrm{~h})$. Microglia $\left(2.5 \times 10^{5}\right.$ cells $\left./ \mathrm{cm}^{2}\right)$ were treated with TRAM-34 $(3 \mu \mathrm{M}$, plus $15 \mathrm{~min}$ pre-treatment) or vehicle (DMSO $0.05 \%$ ). For invasion, GL-15 cells were plated at a density of $7 \times 10^{3} \mathrm{cells} / \mathrm{cm}^{2}$ on matrigel-coated transwells ( $8 \mu \mathrm{m}$ pore size), treated with $100 \mathrm{nM} \mathrm{CXCL12}$ in the presence or in the absence of TRAM-34 $(5 \mu \mathrm{M}, 15 \mathrm{~min}$ pre-treatment), and assayed for $18 \mathrm{~h}$.

Phagocytosis. Microglial cells were seeded on poly-L-lysine-treated $10 \mathrm{~mm}$ glass coverslips $\left(7 \times 10^{4}\right.$ cells). Control medium or medium conditioned for $24 \mathrm{~h}$ with GL-15 or MZC cells was added for $18 \mathrm{~h}$ to microglia in the presence or in the absence of TRAM-34 $(5 \mu \mathrm{M}, 15 \mathrm{~min}$ pre-treatment). Positive controls were obtained by treating microglia with LPS $(500 \mathrm{ng} / \mathrm{ml})$. Medium was then removed, $0.05 \%$ (corresponding to $1.8 \times 10^{7}$ spheres $/ \mathrm{ml}$ ) red fluorescent FluoSpheres were added for $1 \mathrm{~h}$ in serum-free medium $(0.1 \% \mathrm{BSA})$, and nuclei were stained by Hoechst. Cells were washed three times with PBS to remove non-phagocytized spheres and fixed in 4\% PFA for 15 min. Phagocytosis was quantified by counting the number of phagocytizing cells (scoring as positive only cells with at least three FluoSpheres to avoid possible false positives due to sphere adhesion to cell surface; see Labuzek et al. $\left.{ }^{57}\right)$ in at least 20 random fields per coverslip $(594 \mathrm{~nm}$, Axioscope 2; Carl Zeiss, Oberkochen, Germany).

Reverse transcription PCR. Total RNA was extracted from GL-15 cells using the RNA miniprep. DNA contamination was removed according to the manufacturer's protocol. Total RNA $(1 \mu \mathrm{g})$ was reverse transcribed using the Thermo Script RT-PCR System protocol, and $150 \mathrm{ng}$ of the reverse transcription product was used as a template for PCR amplification. Primer sequences were as follows: KCa3.1, 5'-TCAATCAAGTCCGCTTCCG-3'; 5'-ATTCTGCTGCAGGTCA TACAGG-3'; human- $\beta$-actin, $5^{\prime}$-TAAGGAGGAGCTGTGCTACG-3'; and $5^{\prime}$-GGAG CAATGATCTTGATCTTC-3',58-60 primers were used at a final concentration of $0.6 \mu \mathrm{M}$ in the PCR reactions. The PCR reaction was as follow: $95^{\circ} \mathrm{C}$ for $10 \mathrm{~min}$, 45 cycles of $94^{\circ} \mathrm{C}$ for $30 \mathrm{~s}, 55^{\circ} \mathrm{C}$ for $30 \mathrm{~s}$ and $68^{\circ} \mathrm{C}$ for 1 min followed by a final extension step at $72^{\circ} \mathrm{C}$ for $10 \mathrm{~min}$. A MJ Mini Thermal Cycler (Biorad, Segrate MI, Italy) was used for all reactions. Amplification product was analyzed on $1.8 \%$ agarose gel stained with ethidium bromide. Densitometric analysis was performed with QuantityOne software (Biorad) and KCa3.1 cDNA levels were normalized for human- $\beta$-actin.

Orthotopic implantation of tumor cells into the brain of SCID mice. Six-week-old male CB17/SCID mice were anesthetized with chloral hydrate $(400 \mathrm{mg} / \mathrm{kg}$, i.p.) and placed in a stereotaxic head frame. Animals were stereotactically injected with $4 \times 10^{5} \mathrm{GL}-15$ : a median incision of $\sim 1 \mathrm{~cm}$ was made, a burr hole was drilled in the skull, and cells were injected $2.5 \mathrm{~mm}$ lateral (right) and $0.5 \mathrm{~mm}$ anterior to the bregma in the right striatum. Cell suspension in PBS $(10 \mu \mathrm{l})$ was injected with a Hamylton syringe at rate of $1 \mu / \mathrm{min}$ at $3 \mathrm{~mm}$ depth. Xenografted mice were treated daily with TRAM-34 (120 mg/Kg i.p.) or the same volume of vehicle (peanut oil). After 5 weeks, animals were sacrificed and brains were isolated.

Brain concentration of TRAM-34. The brains of mice treated for 5 weeks with TRAM-34 or vehicle were homogenized and purified using C18 solid phase extraction cartridges. Eluted fractions corresponding to TRAM-34 were dried under nitrogen and reconstituted in acetonitrile. LC/MS analysis was performed using a Hewlett-Packard 1100 series HPLC stack (Hewlett-Packard; now Agilent Technologies, Santa Clara, CA, USA) equipped with a Merck KGaA RT 250-4 LiChrosorb RP-18 column (EMD Chemicals, Gibbstown, NJ, USA) interfaced to a Finnigan LCQ Classic MS (Thermo Electron, now Thermo Fisher Scientific, Waltham, MA, USA). The mobile phase consisted of acetonitrile and water, both containing $0.2 \%$ formic acid. With a flow rate of $1.0 \mathrm{ml} / \mathrm{min}$, the gradient was ramped from $20 / 80$ to $70 / 30$ in $5 \mathrm{~min}$, then to $80 / 20$ over $11 \mathrm{~min}$, to $5 / 95$ un to $16.5 \mathrm{~min}$, and finally back to $80 / 20$ up to $38 \mathrm{~min}$. With the column temperature maintained at $30^{\circ} \mathrm{C}$, TRAM-34 eluted at $14.4 \mathrm{~min}$ and was detected by a variable wavelength detector set to $190 \mathrm{~nm}$ and the MS in series. Using electrospray ionization/ion trap MS, TRAM-34 was quantified by its base peak of $277 \mathrm{~m} / \mathrm{z}$ 
(2-chlorotrityl fragment) and concentrations were calculated with a 5-point calibration curve from $25 \mathrm{nmol} / \mathrm{l}$ to $2.5 \mu \mathrm{mol} / \mathrm{l}$.

Immunofluorescence. Coronal brain sections $(10 \mu \mathrm{m})$ were washed in PBS, blocked ( $3 \%$ goat serum in $0.3 \%$ Triton $\mathrm{X}-100$ ) for $1 \mathrm{~h}$ at RT, and incubated overnight at $4{ }^{\circ} \mathrm{C}$ with specific antibodies diluted in PBS containing $1 \%$ goat serum and $0.1 \%$ Triton $\mathrm{X}-100$. After several washes, sections were stained with the fluorophore-conjugated antibody and Hoechst for nuclei visualization and analyzed using a fluorescence microscope.

Image acquisition and data analysis. The effects of TRAM-34 treatment on the extent of human cell infiltration, reactive astrocytosis, and microglial activation in the brain parenchyma of tumor-xenografted animals were evaluated by fluorescence microscopy. Images of fluorescence derived from anti-human nuclei, GFAP or CD68 Abs were digitized using a CooISNAP camera (Photometrics) coupled to a ECLIPSE Ti-S microscope (Nikon) and processed using MetaMorph 7.6.5.0 image analysis software (Molecular Device). Slices were scanned by consecutive fields of vision ( $\times 10$ objective lens) to build a single image per section. Data were expressed as area occupied by fluorescent cells versus total slice area (by converting pixel to $\mu \mathrm{m}^{2}$ ); this pixel-based method was chosen to evaluate the large numbers of cells intensely stained for human nuclei and the irregularly shaped astrocytes and activated microglia. For comparison between different treatments, at least 12 coronal sections per brain, $1.5 \mathrm{~mm}$ around the point of injection were analyzed. To calculate the maximal distance reached by tumor cells on the sagittal axis, we measured the distance between the first and the last slices positively stained for human nuclei.

Statistical analysis. All data are the means \pm S.E.M. of at least four experiments. Statistical significance was determined by Student's $t$-test or one-way ANOVA after checking for data normality.

\section{Conflict of Interest}

The authors declare no conflict of interest.

Acknowledgements. We thank Dr Flavia Trettel for suggestions. This work was funded by Ministero dell'Istruzione, dell'Università e della Ricerca, Programmi di Ricerca Scientifica di Rilevante Interesse Nazionale 2009 (2009SX72KB to C Li), Associazione Italiana per la Ricerca sul Cancro (IG 12774, to CLi) and National Institute of Health (GM076063 to HW).

1. Giese A, Bjerkvig R, Berens ME, Westphal M. Cost of migration: invasion of malignant gliomas and implications for treatment. J Clin Oncol 2003; 21: 1624-1636.

2. Wen PY, Kesari S. Malignant gliomas in adults. N Engl J Med 2008; 359: 492-507.

3. Markovic DS, Glass R, Synowitz M, Nv Rooijen, Kettenmann H. Microglia stimulate the invasiveness of glioma cells by increasing the activity of metalloprotease-2. J Neuropathol Exp Neurol 2005; 64: 754-762.

4. Wesolowska A, Kwiatkowska A, Slomnicki L, Dembinski M, Master A, Sliwa M et al. Microglia-derived TGF-beta as an important regulator of glioblastoma invasion-an inhibition of TGF-beta-dependent effects by shRNA against human TGF-beta type II receptor. Oncogene 2008; 27: 918-930.

5. Charles NA, Holland EC, Gilbertson R, Glass R, Kettenmann H. The brain tumor microenvironment. Glia 2012; 60: 502-514.

6. Takano T, Lin JHC, Arcuino G, Gao Q, Yang J, Nedergaard M. Glutamate release promotes growth of malignant gliomas. Nature Medicine 2001; 7: 1010-1015.

7. El Andaloussi A, Lesniak MS. CD4 + CD25 + FoxP3 + T-cell infiltration and heme oxygenase-1 expression correlate with tumor grade in human gliomas. J Neurooncol 2007 83: $145-152$

8. Coniglio SJ, Eugenin E, Dobrenis K, Stanley ER, West BL, Symons MH et al. Microglial stimulation of glioblastoma invasion involves epidermal growth factor receptor (EGFR) and colony stimulating factor 1 receptor (CSF-1R) signaling. Mol Med 2012; 18: 519-527.

9. Rempel SA, Dudas S, Ge S, Gutiérrez JA. Identification and localization of the cytokine SDF1 and its receptor, CXC chemokine receptor 4, to regions of necrosis and angiogenesis in human glioblastoma. Clin Cancer Res 2000; 6: 102-111.

10. Rubin JB, Kung AL, Klein RS, Chan JA, Sun Y, Schmidt $\mathrm{K}$ et al. A small-molecule antagonist of CXCR4 inhibits intracranial growth of primary brain tumors. Proc Natl Acad Sci USA 2003; 100: 13513-13518.

11. Bian XW, Yang SX, Chen JH, Ping YF, Zhou XD, Wang QL et al. Preferential expression of chemokine receptor CXCR4 by highly malignant human gliomas and its association with poor patient survival. Neurosurgery 2007; 61: 570-578.
12. Zagzag D, Esencay M, Mendez O, Yee H, Smirnova I, Huang $Y$ et al. Hypoxia- and vascular endothelial growth factor-induced stromal cell-derived factor-1alpha/CXCR4 expression in glioblastomas: one plausible explanation of Scherer's structures. Am J Pathol 2008; 173: 545-560

13. Sciaccaluga M, Fioretti B, Catacuzzeno L, Pagani F, Bertollini C, Rosito $M$ et al. CXCL12-induced glioblastoma cell migration requires intermediate conductance $\mathrm{Ca} 2+$ -activated K + channel activity. Am J Physiol Cell Physiol 2010; 299: C175-C184.

14. Kunzelmann K. Ion channels and cancer. J Membr Biol 2005; 205: 159-173.

15. Arcangeli A, Crociani O, Lastraioli E, Masi A, Pillozzi S, Becchetti A. Targeting ion channels in cancer: a novel frontier in antineoplastic therapy. Curr Med Chem 2009; 16: 66-93.

16. Cuddapah VA, Sontheimer $\mathrm{H}$. Ion channels and transporters [corrected] in cancer. 2. Ion channels and the control of cancer cell migration. Am J Physiol Cell Physiol 2011; 301: C541-C549.

17. Cuddapah VA, Turner KL, Seifert S, Sontheimer H. Bradykinin-induced chemotaxis of human gliomas requires the activation of KCa3.1 and CIC-3. J Neurosci 2013; 33: 1427-1440.

18. Ifuku M, Färber $\mathrm{K}$, Okuno $\mathrm{Y}$, Yamakawa $\mathrm{Y}$, Miyamoto $\mathrm{T}$, Nolte $\mathrm{C}$ et al. Bradykinin-induced microglial migration mediated by $\mathrm{B} 1$-bradykinin receptors depends on $\mathrm{Ca} 2+$ influx via reverse-mode activity of the $\mathrm{Na}+/ \mathrm{Ca} 2+$ exchanger. J Neurosci 2007; 27: 13065-13073

19. Ishii TM, Silvia C, Hirschberg B, Bond CT, Adelman JP, Maylie J. A human intermediate conductance calcium-activated potassium channel. Proc Natl Acad Sci USA 1997; 94: 11651-11656

20. Fioretti B, Castigli E, Calzuola I, Harper AA, Franciolini F, Catacuzzeno L. NPPB block of the intermediate-conductance Ca2 + -activated K + channel. Eur J Pharmacol 2004; 497: 1-6.

21. Fioretti B, Castigli E, Micheli MR, Bova R, Sciaccaluga M, Franciolini F et al. Expression and modulation of the intermediate- conductance $\mathrm{Ca} 2+$-activated $\mathrm{K}+$ channel in glioblastoma GL-15 cells. Cell Physiol Biochem 2006; 18: 47-56.

22. Weaver AK, Bomben VC, Sontheimer H. Expression and function of calcium-activated potassium channels in human glioma cells. Glia 2006; 54 : 223-233.

23. Khalid MH, Shibata S, Hiura T. Effects of clotrimazole on the growth, morphological characteristics, and cisplatin sensitivity of human glioblastoma cells in vitro. J Neurosurg 1999; 90: 918-927.

24. Liu H, Li Y, Raisch KP. Clotrimazole induces a late G1 cell cycle arrest and sensitizes glioblastoma cells to radiation in vitro. Anticancer Drugs 2010; 21: 841-849.

25. Khalid MH, Tokunaga Y, Caputy AJ, Walters E. Inhibition of tumor growth and prolonged survival of rats with intracranial gliomas following administration of clotrimazole. J Neurosurg 2005; 103: 79-86

26. Wulff $\mathrm{H}$, Miller MJ, Hansel W, Grissmer S, Cahalan MD, Chandy KG. Design of a potent and selective inhibitor of the intermediate-conductance $\mathrm{Ca} 2+$-activated $\mathrm{K}+$ channel, IKCa1: a potential immunosuppressant. Proc Natl Acad Sci USA 2000; 97: 8151-8156.

27. Köhler R, Wulff H, Eichler I, Kneifel M, Neumann D, Grgic I et al. Blockade of the intermediate-conductance calcium-activated potassium channel as a new therapeutic strategy for restenosis. Circulation 2003; 108: 1119-1125.

28. Chen YJ, Raman G, Bodendiek S, O'Donnell ME, Wulff H. The KCa3.1 blocker TRAM-34 reduces infarction and neurological deficit in a rat model of ischemia/reperfusion stroke. J Cereb Blood Flow Metab 2011; 31: 2363-2374.

29. Reich EP, Cui L, Yang L, Pugliese-Sivo C, Golovko A, Petro M et al. Blocking ion channel KCNN4 alleviates the symptoms of experimental autoimmune encephalomyelitis in mice. Eur J Immunol 2005; 35: 1027-1036.

30. Grgic I, Heinau P, Si H, Brakemeier S, Hoyer J, Köhler R. Selective blockade of the intermediate-conductance $\mathrm{Ca} 2+$-activated $\mathrm{K}+$ channel suppresses proliferation of microvascular and macrovascular endothelial cells and angiogenesis in vivo. Arterioscler Thromb Vasc Biol 2005; 25: 704-709.

31. Toyama K, Wulff H, Chandy KG, Azam P, Raman G, Saito T et al. The intermediateconductance calcium-activated potassium channel $\mathrm{KCa} 3.1$ contributes to atherogenesis in mice and humans. J Clin Invest 2008; 118: 3025-3037.

32. Girodet PO, Ozier A, Carvalho G, llina O, Ousova O, Gadeau AP et al. Ca2 + -Activated $\mathrm{K}+$ Channel-3.1 Blocker TRAM-34 Attenuates Airway Remodeling and Eosinophilia in a Murine Asthma Model. Am J Respir Cell Mol Biol 2013; 48: 212-219.

33. Guillamo JS, Lisovoski F, Christov C, Le Guérinel C, Defer GL, Peschanski M et al. Migration pathways of human glioblastoma cells xenografted into the immunosuppressed rat brain. J Neurooncol 2001: 52: 205-215.

34. Hoelzinger DB, Demuth T, Berens ME. Autocrine factors that sustain glioma invasion and paracrine biology in the brain microenvironment. J Natl Cancer Inst 2007; 99: 1583-1593.

35. Kroonen J, Nassen J, Boulanger YG, Provenzano F, Capraro V, Bours V et al. Human glioblastoma-initiating cells invade specifically the subventricular zones and olfactory bulbs of mice after striatal injection. Int J Cancer 2011; 129: 574-585.

36. Kaushal V, Koeberle PD, Wang Y, Schlichter LC. The Ca2 + -activated K + channel $\mathrm{KCNN} 4 / \mathrm{KCa} 3.1$ contributes to microglia activation and nitric oxide-dependent neurodegeneration. J Neurosci 2007; 27: 234-244.

37. Marrelli SP, Eckmann MS, Hunte MS. Role of endothelial intermediate conductance KCa channels in cerebral EDHF-mediated dilations. Am J Physiol Heart Circ Physiol 2003; 285 . H1590-H1599. 
38. Cronin CA, Gluba W, Scrable H. The lac operator-repressor system is functional in the mouse. Genes Dev 2001; 15: 1506-1517.

39. Wu JD, Hsueh HC, Huang WT, Liu HS, Leung HW, Ho YR et al. The inducible lactose operator-repressor system is functional in the whole animal. DNA Cell Biol 1997; 16: $17-22$.

40. Lee J, Borboa AK, Baird A, Eliceiri BP. Non-invasive quantification of brain tumor-induced astrogliosis. BMC Neurosci 2011; 12: 19.

41. Ruggieri P, Mangini G, Fioretti B, Catacuzzeno L, Puca R, Ponti D et al. The inhibition of $\mathrm{KCa} 3.1$ channels activity reduces cell motility in glioblastoma derived cancer stem cells. Plos ONE 2012; 7: e47825.

42. Watkins $\mathrm{S}$, Sontheimer $\mathrm{H}$. Hydrodynamic cellular volume changes enable glioma cell invasion. J Neurosci 2011; 31: 17250-17259.

43. Catacuzzeno L, Aiello F, Fioretti B, Sforna L, Castigli E, Ruggieri P et al. Serum-activated K and $\mathrm{Cl}$ currents underlay U87-MG glioblastoma cell migration. J Cell Physiol 2011; 226 1926-1933.

44. Iskandar F, Abdullaev IF, Rudkouskaya A, Mongin AA, Kuo YH. Calcium-activated potassium channels BK and IK1 are functionally expressed in human gliomas but do not regulate cell proliferation. PLOS ONE 2010; 5: e12304.

45. Maezawa I, Jenkins DP, Jin BE, Wulff $H$. Microglial $\mathrm{KCa} 3.1$ channels as potential therapeutic target for Alzheimer's disease. IAD 2011; 2012: 868972.

46. Schilling $T$, Stock $C$, Schwab A, Eder $C$. Functional importance of $\mathrm{Ca} 2+$-activated $\mathrm{K}+$ channels for lysophosphatidic acid-induced microglial migration. Eur J Neurosci 2004; 19 1469-1474.

47. Khanna R, Roy L, Zhu X, Schlichter LC. K + channels and the microglial respiratory burst. Am J Physiol Cell Physiol 2001; 280: C796-C806.

48. Hussain SF, Yang D, Suki D, Aldape K, Grimm E, Heimberger AB. The role of human glioma-infiltrating microglia/macrophages in mediating antitumor immune responses. Neuro Oncol 2006; 8: 261-279.

49. Schartner JM, Hagar AR, Van Handel M, Zhang L, Nadkarni N, Badie B. Impaired capacity for upregulation of MHC class II in tumor-associated microglia. Glia 2005; 51: 279-285.

50. Li W, Graeber MB. The molecular profile of microglia under the influence of glioma. Neuro Oncol 2012; 14: 958-978.

51. Wu A, Wei J, Kong LY, Wang Y, Priebe W, Quiao W et al. Glioma cancer stem cells induce immunosuppressive macrophages/microglia. Neuro Oncol 2010; 12: 1113-1125.
52. Komohara $Y$, Ohnishi $K$, Kuratsu J, Takeya M. Possible involvement of the M2 anti-inflammatory macrophage phenotype in growth of human gliomas. J Pathol 2008; 216: $15-24$.

53. Bouhy D, Ghasemlou N, Lively S. Inhibition of the Ca2 + - dependent $\mathrm{K}+$ channel $\mathrm{KCNN} 4 / \mathrm{KCa} 3.1$, improves tissue protection and locomotor recovery after spinal cord injury J Neurosci 2011; 31: 16298-16308.

54. Becher OJ, Hambardzumyan D, Fomchenko El, Momota H, Mainwaring L, Bleau AM et al. Gli activity correlates with tumor grade in platelet-derived growth factor-induced gliomas. Cancer Res 2008; 68: 2241-2249.

55. Lauro C, Cipriani R, Catalano M, Trettel F, Chece G, Brusadin V et al. Adenosine A1 receptors and microglial cells mediate $\mathrm{CX} 3 \mathrm{CL} 1$-induced protection of hippocampal neurons against Glu-induced death. Neuropsychopharmacol 2010; 35: 1550-1559.

56. Fioretti B, Catacuzzeno L, Sforna L, Aiello F, Pagani F, Ragozzino D et al. Histamine hyperpolarizes human glioblastoma cells by activating the intermediate-conductance Ca-activated K channel. Am J Physiol Cell Physiol 2009; 297: C102-C110.

57. Labuzek K, Liber S, Gabryel B, Adamczyk J, Okopien B. Metformin increases phagocytosis and acidifies lysosomal/endosomal compartments in AMPK-dependent manner in rat primary microglia. Naunyn Schmied. Arch Pharmacol 2010; 381: 171-186.

58. Carignani C, Roncarati R, Rimini R, Terstappen GC. Pharmacological and molecular characterisation of SK3 channels in the TE671 human medulloblastoma cell line. Brain Res 2002; 939: 11-18

59. Liu X, Chang Y, Reinhart PH, Sontheimer H, Chang Y. Cloning and characterization of glioma BK, a novel BK channel isoform highly expressed in human glioma cells. J Neurosci 2002; 22: 1840-1849.

60. Logsdon NJ, Kang J, Togo JA, Christian EP, Aiyar J. A novel gene, hKCa4, encodes the calcium-activated potassium channel in human T lymphocytes. J Biol Chem 1997; 272: 32723-32726.

(c) (i) $(\theta)$ Cell Death and Disease is an open-access journal published by Nature Publishing Group. This work is licensed under a Creative Commons Attribution-NonCommercialNoDerivs 3.0 Unported License. To view a copy of this license, visit http://creativecommons.org/licenses/by-nc-nd/3.0/ 